\title{
Élaboration de films minces de tellurure de bismuth par voie chimique
}

\author{
L. Scidone, C. Boulanger, J.M. Lecuire et S. Diliberto \\ Laboratoire d'Électrochimie des Matériaux UMR CNRS 7555, Université de Metz, \\ 1 Bd. Arago, Technopôle, CP 87811, 57078 Metz Cedex 3, France
}

\begin{abstract}
Résumé. La miniaturisation de modules Peltier passe par la recherche de techniques de synthèse appropriées pour l'élaboration de film minces d'alliages de matériaux thermoélectriques. Les procédés de synthèse couramment rencontrés sont basés sur différents principes présentant de nombreux inconvénients techniques. Une technique plus simple d'élaboration par déposition chimique de ces matériaux de type $\mathrm{Bi}_{2} \mathrm{Te}_{3}$ sous forme de films minces a été étudiée. Les dépôts ont été effectués à partir de solutions de $\mathrm{Te}^{\mathrm{IV}}$ et $\mathrm{Bi}^{\mathrm{III}}$ dissous en milieu nitrique à température ambiante. La réduction de ces cations a été envisagée selon deux voies. D'une part par l'utilisation de différents réducteurs solubles et d'autre part par action de métaux purs. L'influence de la concentration en cations métalliques et le rapport $\mathrm{Bi}^{\mathrm{III}} / \mathrm{Te}^{\mathrm{IV}}$ des solutions ont été étudiés. Les produits de synthèse ont été identifiés par diffraction des rayons $\mathrm{X}$, l'aspect des films a été observé par microscopie électronique à balayage. Enfin, la cinétique de croissance d'un film thermoélectrique a été étudiée par suivi gravimétrique pour des temps de déposition assez longs $(>30 \mathrm{~min}$ ) et sur microbalance à quartz pour des temps de déposition courts $(<30 \mathrm{~min})$.
\end{abstract}

\section{INTRODUCTION}

L'élaboration d'alliages de la famille des chalcogénures notamment celles des tellurures de bismuth constitue un enjeu industriel important de part leurs propriétés thermoélectriques.

Ainsi, la première méthode de préparation de ce composé a été réalisée en 1951 par réaction à l'état solide [1]. Depuis d'autres principes de synthèse ont été développés, basés sur des techniques conventionnelles de cristallisation directe (méthode Czochralski [2]) ou de déplacement de zone fondue (méthode Bridgmann [3]). Ces matériaux sous forme de films sont également élaborés au moyen de techniques d'évaporation [4], de flash évaporation [5], de pulvérisation cathodique [6], de jet moléculaire [7] ou par MOCVD [8]. Il est à noter que ces différentes techniques sont difficiles à mettre en œuvre et conduisent à des surfaces de déposition restreintes. C'est pourquoi une autre méthode a été développée basée sur le principe de l'électrodéposition $[9,10,11,12]$. Cette technique permet de déposer sur des plus grandes surfaces et la stæechiométrie du composé électrodéposé peut être maîtrisée par le biais des paramètres chimiques et électrochimiques. Cependant, un impératif technique important impose la répartition régulière des lignes de champs et l'intervention d'un substrat conducteur. Ces contraintes pourraient ainsi être évitées en mettant au point une alternative supplémentaire d'élaboration du matériau par la voie de la chimie douce permettant ainsi de diminuer le coût de fabrication, d'élargir le panel des types de supports notamment des non conducteurs. 
Dans cet article, nous présentons les premiers résultats concernant l'élaboration de films minces de tellurure de bismuth par réduction chimique. Deux méthodes sont considérées: d'une part l'intervention d'un réducteur soluble en solution et d'autre part l'intervention d'un support réducteur métallique.

\section{PARTIE EXPÉRIMENTALE}

Les produits utilisés lors de la préparation du bain sont les suivants : (tableau 1).

Tableau 1. Réactifs, paramètres physiques et fonction des produits du bain electroless.

\begin{tabular}{ccc}
\hline Réactif et paramètre physique & Fonction & Concentration (mol/l) \\
\hline $\mathrm{Te}^{\circ}$ & Source des ions $\mathrm{Te}^{\mathrm{IV}}$ & $2.10^{-2}$ \\
$\mathrm{Bi}_{\left(\mathrm{NO}_{3}\right)_{3}, 5 \mathrm{H} 2 \mathrm{O}}$ & Source des ions Bi ${ }^{\mathrm{III}}$ & $3.33 .10^{-3}-2.10^{-2}$ \\
$\mathrm{HNO}_{3}$ & électrolyte & 1 \\
$\mathrm{pH}$ & 0 & \\
Température & $19-21^{\circ} \mathrm{C}$ \\
\hline
\end{tabular}

Les couples d'oxydoréduction engagés dans le bain de déposition sont représentés dans le tableau 2.

Tableau 2. Couples redox engageant les cations présents dans le bain electroless.

\begin{tabular}{cccc}
\hline $\begin{array}{c}\text { Couples redox } \\
\text { considérés }\end{array}$ & $\begin{array}{c}\text { Potentiel redox } \\
\text { standart, } \mathrm{E}^{\circ} \\
\left(\mathrm{V} / \mathrm{ENH}_{2}\right)\end{array}$ & $\begin{array}{c}\text { Masse moléculaire } \\
\left({\left.\mathrm{g} . \mathrm{mol}^{-1}\right)}^{-1}\right.\end{array}$ & $\begin{array}{c}\text { Nombre d'électrons } \\
\text { échangés }\end{array}$ \\
\hline $\mathrm{Bi}^{+\mathrm{III}}, \mathrm{Te}^{+\mathrm{IV}} / \mathrm{Bi}_{2} \mathrm{Te}_{3}$ & 0.54 & 800.76 & 18 \\
$\mathrm{Te}^{+\mathrm{IV}} / \mathrm{Te}^{\circ}$ & 0.47 & 127.60 & 4 \\
$\mathrm{Bi}^{+\mathrm{III}} / \mathrm{Bi}^{\circ}$ & 0.23 & 208.98 & 3 \\
\hline
\end{tabular}

Les solutions sont obtenues par dissolution de $\mathrm{Bi}(\mathrm{NO} 3) 3,5 \mathrm{H} 2 \mathrm{O}$ et de $\mathrm{Te}^{\circ}$ dans l'acide nitrique $7 \mathrm{M}$. Celles-ci sont alors diluées à l'eau distillée afin d'obtenir les concentrations en cations et le $\mathrm{pH}$ souhaités. Les dépôts sont effectués à température ambiante.

Dans le cas des réducteurs solubles, la réaction est effectuée dans un premier temps sous agitation jusqu'à dissolution totale du réducteur, puis la solution est laissée au repos pendant une heure. Les produits de synthèse sont filtrés, lavés à l'acide nitrique $0.1 \mathrm{M}$ puis à l'eau distillée. La réaction sur support réducteur métallique est effectuée en plongeant directement le substrat dans la solution pendant une heure en l'absence d'agitation, les produits de synthèse sont ensuite lavés à l'acide nitrique $0.1 \mathrm{M}$ puis à l'eau distillée. Les produits de synthèse ont été caractérisés par DRX (spectromètre INEL XRG 2500 avec un détecteur CPS 120), enfin, la cinétique de croissance de dépôt a été étudiée par gravimétrie et par suivi sur microbalance à quartz (MAXTEK PM710). 


\section{RÉSULTATS ET DISCUSSION}

\subsection{Par réaction d'un réducteur soluble}

Les réducteurs ont été sélectionnés en fonction de leur potentiel d'oxydoréduction.

Les quantités de réducteurs ajoutés sont stœchiométriques par rapport aux cations considérant la réaction de réduction suivante :

$$
2 \mathrm{Bi}^{3+}+3 \mathrm{HTeO}_{2}^{+}+18 \mathrm{e}^{-}+9 \mathrm{H}^{+} \rightarrow \mathrm{Bi}_{2} \mathrm{Te}_{3} \downarrow+6 \mathrm{H}_{2} \mathrm{O} .
$$

Les réducteurs solubles testés ont été : dihydrogénohypophosphite de sodium $\left(\mathrm{NaH}_{2} \mathrm{PO}_{2}\right)$, l'acide oxalique $\left(\mathrm{C}_{2} \mathrm{H}_{2} \mathrm{O}_{4}\right)$, l'hydrazine $\left(\mathrm{N}_{2} \mathrm{H}_{4}\right)$, le sulfite de sodium $\left(\mathrm{Na}_{2} \mathrm{SO}_{3}\right)$, le chlorure d'hydroxylamine $\left(\mathrm{H}_{3} \mathrm{NO} . \mathrm{HCl}\right)$ et le thiosulfate de sodium $\left(\mathrm{Na}_{2} \mathrm{~S}_{2} \mathrm{O}_{3}\right)$.

Seuls, le dihydrogénohypophosphite de sodium et le thiosulfate de sodium ont conduit à la formation d'un précipité noir. La caractérisation de ces produits par diffraction des rayons $\mathrm{X}$ montre dans les deux cas qu'il s'agit de $\mathrm{Te}^{\circ}$ pur cristallisé (figure 1).

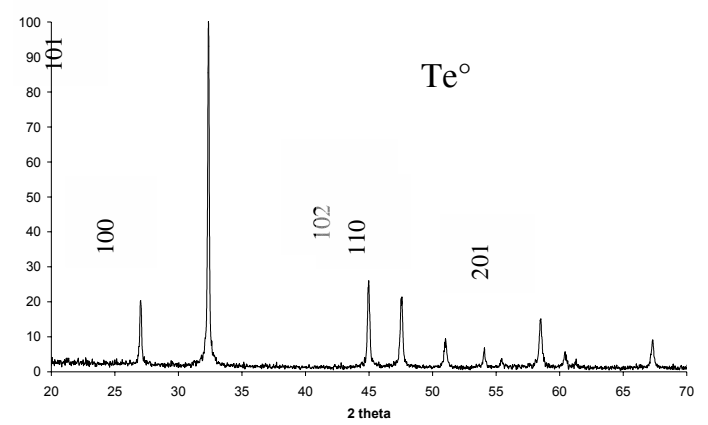

Figure 1. Diffractogramme effectué sur les produits de réaction des réducteurs $\mathrm{NaH}_{2} \mathrm{PO}_{2}$ et de $\mathrm{Na}_{2} \mathrm{~S}_{2} \mathrm{O}_{3}$ dans un bain $\mathrm{Bi}^{\mathrm{III}} / \mathrm{Te}^{\mathrm{IV}}=2 / 3,\left[\mathrm{Te}^{\mathrm{IV}}\right]=10^{-2} \mathrm{M} \cdot \lambda_{\mathrm{Co}}=1.78897 \AA$.

Il n'a pas été montré la possibilité de synthétiser un alliage de type tellurure de bismuth par réduction chimique via un réducteur soluble mais uniquement du tellure élémentaire sous forme de grains très fins se formant dans tout le volume de la solution.

\subsection{Par réaction sur support réducteur}

\subsubsection{Différents supports réducteurs}

Les supports réducteurs testés ont été : nickel, étain, cuivre, fer, zinc.

$\mathrm{La}$ composition des bains de déposition est d'un rapport $2 / 3$ en cations $\mathrm{Bi}{ }^{\mathrm{III}} / \mathrm{Te}^{\mathrm{IV}}$ avec une concentration $2.10^{-2} \mathrm{M}$ en $\mathrm{Te}^{\mathrm{IV}}$ en milieu nitrique $1 \mathrm{M}$ à température ambiante pour des temps de dépôt d'une heure.

Pour le fer et le zinc, la corrosion par le milieu acide ne permet pas d'envisager le dépôt de films minces de tellurure de bismuth. Dans le cas des autres supports, des films se forment rapidement à la surface des substrats. Les analyses DRX de ces films sont présentés sur les figures 2, 3 et 4. 

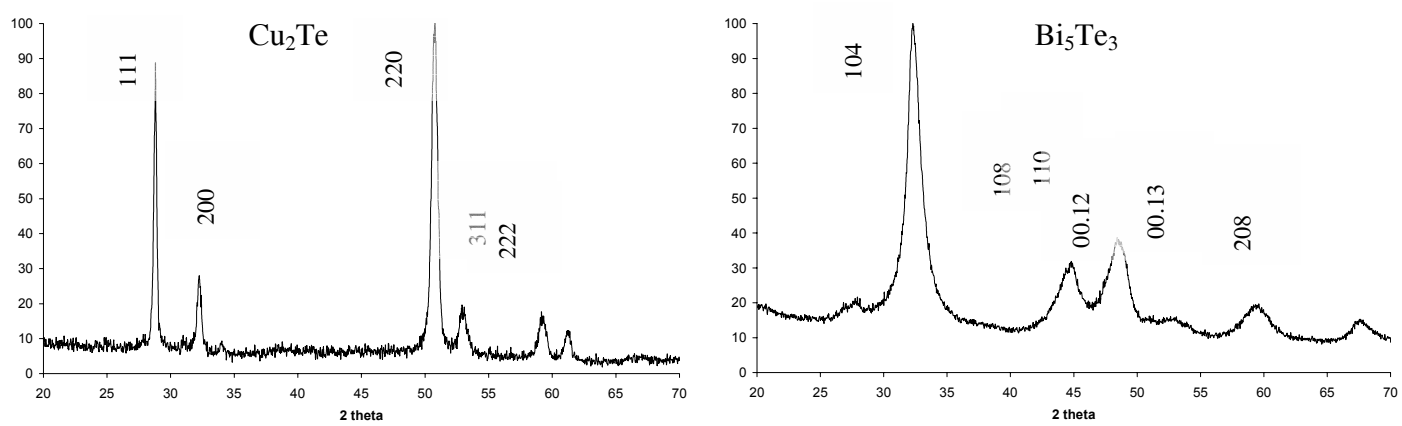

Figure 2. Diffractogramme du produit de réaction sur Figure 3. Diffractogramme du produit de réaction sur $\mathrm{Cu}^{\circ}$ (indexé par fiche ASTM 19-116). $\lambda_{\mathrm{Co}}=1.78897 \AA . \quad \mathrm{Sn}^{\circ}$ (indexé par fiche ASTM 19-176). $\lambda_{\mathrm{Co}}=1.78897 \AA$.

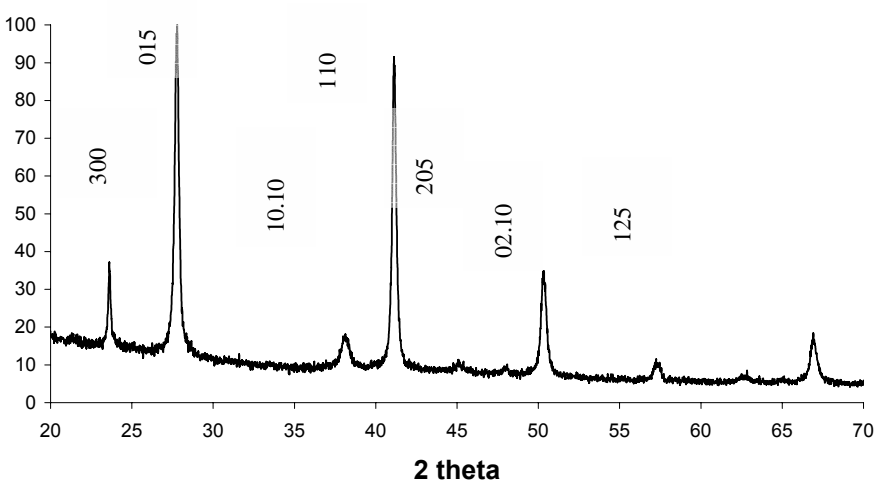

Figure 4. Diffractogramme du produit de réaction sur $\mathrm{Ni}^{\circ}$ (indexé par fiche $\mathrm{ASTM} 15-863$ ). $\lambda_{\mathrm{Cu}}=1.54056 \AA$.

Les diffractogrammes montrent qu'avec le cuivre comme support réducteur, le produit de synthèse n'est pas un tellurure de bismuth. Le produit formé est $\mathrm{Cu}_{2}$ Te qui se forme selon la réaction suivante:

$$
\mathrm{HTeO}_{2}^{+}+4 \mathrm{Cu}^{\circ}+3 \mathrm{H}^{+} \rightarrow \mathrm{Cu}_{2} \mathrm{Te} \downarrow+2 \mathrm{Cu}^{2+}+2 \mathrm{H}_{2} \mathrm{O}
$$

Un produit noir et pulvérulent identifié par diffraction des rayons $\mathrm{X}$ comme étant un alliage de type $\mathrm{Bi}_{5} \mathrm{Te}_{3}$ très mal cristallisé est obtenu sur l'étain. Dans le cas du nickel, le diffractogramme montre que le composé formé a une structure de type $\mathrm{Bi}_{2} \mathrm{Te}_{3}$ cristallisé sous forme d'une maille hexagonale avec une orientation préférentielle pour le plan (110). Le nickel a donc été retenu pour la suite de l'étude.

\subsection{2 Étude sur support réducteur $N i^{\circ}$.}

Les solutions de déposition ont une concentration fixe égale à $2.10^{-2} \mathrm{M}$ en $\mathrm{Te}^{\mathrm{IV}}$ et variable en $\mathrm{Bi}^{\mathrm{III}}$ en milieu nitrique $1 \mathrm{M}$. Les rapports en cations $\mathrm{Bi}^{\mathrm{III}} / \mathrm{Te}^{\mathrm{IV}}$ sont $1 / 6,1 / 3,2 / 3$ et 1 .

Les diffractogrammes effectués sur les produits de réaction sur nickel sont représentés figure 5. 


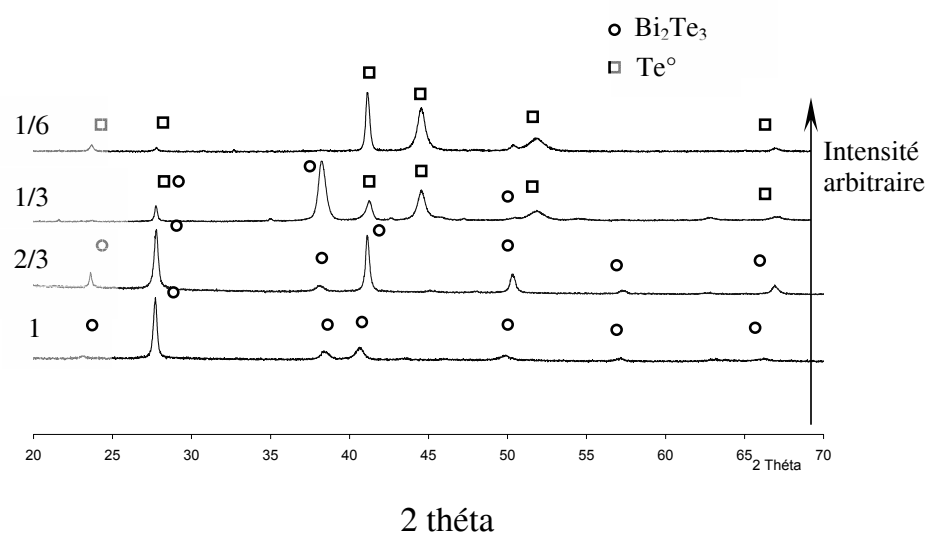

Figure 5. Diffractogrammes obtenus par réaction sur $\mathrm{Ni}^{\circ}$ avec des bains de concentration $2.10^{-2} \mathrm{M}$ en $\mathrm{Te}^{\mathrm{IV}}$ et différents rapports $\mathrm{Bi}^{\mathrm{III}} / \mathrm{Te}^{\mathrm{IV}} . \lambda_{\mathrm{Cu}}=1.54056 \AA$.

Pour des compositions de bain ayant des rapports BiIII/TeIV égaux à 1 et $2 / 3$, les diffractogrammes obtenus sont caractéristiques d'alliages de type tellurure de bismuth, en effet les raies ont pu être indexées par la fiche ASTM n ${ }^{\circ} 15-863$. Les rapports d'intensités de raies sont respectés pour le rapport 1, par contre dans le cas du rapport 2/3, l'augmentation de l'intensité de la raie (110) met en évidence une orientation préférentielle du film.

Le rapport $\mathrm{Bi}^{\mathrm{III}} / \mathrm{Te}^{\mathrm{IV}}$ dans la solution a une incidence directe sur la cristallisation du binaire $\mathrm{Bi}_{2} \mathrm{Te}_{3}$. Pour le rapport $\mathrm{Bi}^{\mathrm{III}} / \mathrm{Te}^{\mathrm{IV}}$ égal à $1 / 3$, les produits obtenus sont du tellure élémentaire et du tellurure de bismuth. Pour ce rapport, le défaut de bismuth du bain ne permet plus la réaction exclusive de réduction de $\mathrm{Te}^{\mathrm{IV}}$ en $\mathrm{Te}^{-\mathrm{II}}$ pour former $\mathrm{Bi}_{2} \mathrm{Te}_{3}$, une partie des cations $\mathrm{Te}^{\mathrm{IV}}$ sont réduits en $\mathrm{Te}^{\circ}$. Pour le rapport $\mathrm{Bi}{ }^{\mathrm{III}} / \mathrm{Te}^{\mathrm{IV}}$ égal à $1 / 6$, le produit obtenu est du tellure élémentaire.

La cinétique de croissance du film a été étudiée par microbalance à quartz (QCM) sur un électrodépôt préalable de $\mathrm{Ni}^{\circ}$ de 2 microns pour les premières minutes de synthèse et par gravimétrie (figure 6) pour des temps de dépôt de plus longue durée. Les mesures par gravimétrie ont été répétées 5 fois.

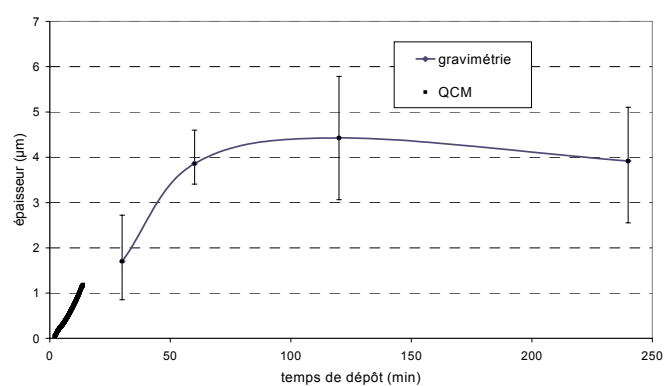

Figure 6. Suivi de la croissance du dépôt par microbalance QCM et par gravimétrie $\left(\left[\mathrm{Te}^{\mathrm{IV}}\right]=2.10^{-2} \mathrm{M}\right.$, $\left.\mathrm{Bi}^{\mathrm{III}} / \mathrm{Te}^{\mathrm{IV}}=2 / 3, \mathrm{HNO}_{3} 1 \mathrm{M}\right)$.

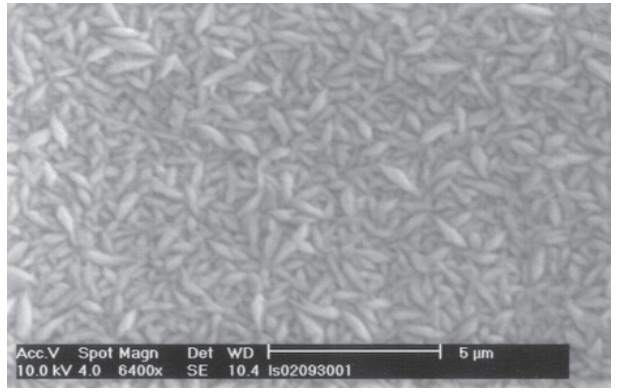

Figure 7. Image en électrons secondaires d'un dépôt obtenu sur $\mathrm{Ni}^{\circ}$ dans un bain de concentration en $\left[\mathrm{Te}^{\mathrm{IV}}\right]=2 \cdot 10^{-2} \mathrm{M}, \mathrm{Bi}^{\mathrm{III}} / \mathrm{Te}^{\mathrm{IV}}=2 / 3, \mathrm{HNO}_{3} 1 \mathrm{M}$.

La cinétique de dépôt est quasiment linéaire pendant la première demi-heure puis diminue jusqu'à être nulle au bout de 2 heures correspondant à un arrêt de la réaction d'oxydoréduction. Ceci est dû à un recouvrement total du support ce qui limite l'épaisseur du dépôt. Il est possible de déterminer la 
contribution de la masse du dépôt formé à partir des variations de masse mesurées expérimentalement et donc de calculer l'épaisseur théorique du dépôt. Dans le cas présent, le film déposé à la stoechiométrie $\mathrm{Bi}_{1.65} \mathrm{Te}_{3.35}$. Son épaisseur théorique est de $5 \mu \mathrm{m}$.

La morphologie des grains a été observée par microscopie électronique à balayage sur un dépôt obtenu sur $\mathrm{Ni}^{\circ}$ (figure 7). L'observation MEB du film déposé met en évidence que le film de type $\mathrm{Bi}_{2} \mathrm{Te}_{3}$ est constitué de grains allongés de $1 \mu \mathrm{m}$ de long sur $0.2-0.3 \mu \mathrm{m}$ de large. Il s'agit vraisemblablement en fait de plaquettes posées selon le plan (110) correspondant à l'axe de croissance du film donc à l'orientation préférentielle observée par diffraction des rayons X.

\section{CONCLUSION}

La synthèse de films de $\mathrm{Bi}_{2} \mathrm{Te}_{3}$ réguliers et homogènes est très facilement réalisable par réaction entre une solution de $\mathrm{Bi}^{\mathrm{III}}$ et $\mathrm{Te}^{\mathrm{IV}}$ sur du nickel métal. L'évolution de la composition de la solution permet d'influencer la stæchiométrie du film et son orientation cristalline ce qui peut éventuellement conduire à des propriétés thermoélectriques différentes. L'inconvénient de cette technique est la limitation de l'épaisseur du film en raison d'un recouvrement total $\mathrm{du} \mathrm{Ni}^{\circ}$ le rendant inaccessible pour permettre la poursuite de la réaction.

\section{Références}

[1] DONGES, Z. Anorg, Allgem. Chem. (1951) 56

[2] N. C. ABRIKOSOV, T. E. SVECHNIKOVA, S. CHIZHEVSKAYA, Neorg. Mat., 14 (1978), 43

[3] HARMON, J. Phys. Chem., 2 (1957) 181

[4] J. PRZYLUSKI, K. BORKOWSKI, Proceeding of the 6th International Conference on Thermoelectrics (1986) 100

[5] C. MACHET, P. LEBON, A. SEPTIER, Le vide-Les couches minces, 211 (1982) 125

[6] Y.SHING, Y. SHANG, A. MIRSHAFFI, L. HAYASHI, S. ROBERTS, J. JOSEFOURIEZ, N. TRAN, J. Vac. Sci. Technol., A1 (1983) 503

[7] E.CHARLES, E. GROUBERT, A. BOYER, J. Materials Sciences Letters, 7 (1988) 579

[8] A. GIANI \& al, Thin Films 303 (1997) 1

[9] P.MAGRI, C. BOULANGER, J. M. LECUIRE, J. Mat Chem., 6(5) (1996) 773

[10] P MAGRI, Thèse de l'université de Metz, 1995

[11] S. MICHEL, Thèse de l'université de Metz, 2003

[12] S. MiCHEL, N. STEIN, M. SCHNEIDER, C. BOUlANGER, J. M. LECUIRE, J. Appl. Electrochem., 33(1) (2003) 23 